Our purpose was to quantify morphological characteristics of extensor digitorum longus (EDL) and soleus (SOL) muscle grafts in female ( $N=$ 8) and male $(N=8)$ rats. Muscles were grafted orthotopically, with the nerve remaining intact, and were studied $\mathbf{5 6}$ days later. The mass of EDL and SOL grafts and control muscles of females was $60 \%$ to $65 \%$ of male values; this difference was directly related to gender differences in body mass. The fiber composition of EDL and SOL grafts did not differ from control, and no gender effects were noted. The mean fiber area (MFA) of control EDL and SOL muscles of females averaged $65 \%$ of male values. The MFA of grafts did not differ due to gender, and averaged $60 \%$ of control value for SOL and $70 \%$ for EDL grafts. We conclude there are no substantial differences in the regenerative capacity of EDL and SOL muscles grafted with the nerve intact.

MUSCLE \& NERVE 8:99-104 1985

\title{
MORPHOLOGY OF STABLE MUSCLE GRAFTS OF RATS: EFFECTS OF GENDER AND MUSCLE TYPE
}

\author{
KATHRYN I. CLARK, MS, and TIMOTHY P. WHITE, PhD
}

Some structural and functional characteristics of stable skeletal muscle grafts in rats remain less than values for control muscle: ${ }^{4,5}$ Facilitation of the reinnervation or revascularization processes during the grafting operation affects the magnitude and type of deficit. ${ }^{7,12}$ Postoperative procedures, such as exercise-conditioning, also have a marked influence on characteristics of muscle grafts. ${ }^{24}$ To address the effects of operative and postoperative interventions, different studies have used female and male rats and have focused on different muscle types. Control extensor digitorum longus (EDL) and soleus (SOL) muscles likely differ with respect to recruitment during standing and locomotion and differ in fiber type ${ }^{1}$ and joint function. ${ }^{16}$ One difference between female and male rats is the ontogenic growth rate and resultant adult body size. ${ }^{15}$ The diurnal secretion patterns

From the Department of Kinesiology, The University of Michigan, Ann Arbor, MI.

Acknowledgments: The authors are grateful to Pedro G. Morales, MD, for assistance with histochemical incubations and Nina Kasper-White for manuscript preparation. The study was supported by National Institutes of Health grants AM29732 and NS17017.

Address reprint requests to Dr. White at the Department of Kinesiology The University of Michigan, 401 Washtenaw Avenue, 1271-B CCRB, Ann Arbor, Ml 48109-2214.

Received for publication December 23, 1983; revised manuscript accepted for publication May 10, 1984.

0148-639X/0802/0099 \$04.00/0

- 1985 John Wiley \& Sons, Inc. for growth hormone and androgens differ between female and male rats, ${ }^{11}$ and may influence the regenerative processes of grafts. A systematic characterization of the morphological profiles of stabilized EDL and SOL muscles grafted orthotopically in female and male rats has not been done.

Our purpose was to characterize the morphology of stable, nerve-intact grafts of SOL and EDL muscles in female and male rats of the same age. We hypothesized that when data were expressed relative to respective control values, graft mass, length, mean fiber area, and fiber composition would not differ due to gender or muscle type.

\section{METHODS}

Female and male Sprague-Dawley rats (Charles River, Kingston, NY) were used in this study. Animals were housed in pairs in metal cages with 10 hours of light and 14 hours of darkness per day. Male and female rats were housed separately, and food (Formulab Chow \#5008, Ralston Purina Company, St. Louis, MO) and water were available ad libitum. Rats were treated in accordance with the Guiding Principles in the Care and Use of Animals approved by the Council of The American Physiological Society.

Grafting surgery was performed at 42 days postpartum. Tetracycline was added to the drinking water of the rats the day before and for 2 days after the grafting operation. Animals were anesthetized with an intraperitoneal injection of sodium pentobarbital ( $35 \mathrm{mg} / \mathrm{kg}$; supplemented as 
required) and nerve-intact grafts were performed on the EDL muscle of one leg and the SOL muscle of the other leg ( $n=6$ rats of each gender). Unoperated contralateral muscles were used for control, as were bilateral unoperated muscles from two additional age-matched animals of each gender.

For grafting of the EDL muscle, the proximal and distal tendons and all blond vessels were severed and the three branches of the nerve were left intact. ${ }^{7}$ The grafting procedure was similar for the SOL muscle, except there is only a single nerve branch which was left intact. Compared to standard grafts in which reinnervation occurs spontaneously, nerve-intact grafts reach a greater mass in a shorter time period. ${ }^{7}$ Qualitatively, the sequence of degenerative and regenerative events does not differ between models. By using the nerve-intact model, our experimental design has focused on factors inherent to the muscles.

The animals węre weighed weekly following grafting. At 56 days postgrafting, animals were anesthetized and muscle lengths were measured from the distal to the proximal ends of the muscle fibers with the ankle and knee joints at $90^{\circ}$. Muscles were removed and weighed. A full transverse section of each muscle was removed from the midpoint and frozen in 2-methylbutane cooled by solid carbon dioxide for histochemical analysis. Transverse sections $10-\mu \mathrm{m}$ thick were cut from the frozen muscle in a cryostat (Ames Company, Elkhart, IN). Sections were incubated for myofibrillar ATPase activity at $\mathrm{pH} 10.3$ and $4.6,{ }^{9}$ and for succinate dehydrogenase activity. ${ }^{20}$ Histochemical sections were projected at $600 \times$. Projections were analyzed for mean fiber area irrespective of fiber type and for composition of different fiber types. ${ }^{3,21}$ The animal was killed with an overdose of sodium pentobarbital. The leg was amputated at the midpoint of the femur, and soft tissues were removed from the leg following 30 minutes immersion in boiling water. The total length of the tibia bone was measured.

Aggregate data are expressed as mean \pm 1 standard error of the mean. Statistical differences were ascertained using a Student's $t$ test modified with the Welch-Apsin convention $(P<0.05$ accepted as significant). This statistic does not require equal numbers of observations in groups and assumes variances to be unknown and not necessarily equal. ${ }^{19}$ Statistical comparisons were made: between grafts and control muscles ncsted within gender and muscle type, and between female and male grafts and control muscles nested within muscle type.

\section{RESULTS}

No significant differences were found in body mass or any of the control variables measured between muscles from unoperated age-matched rats and muscles that were contralateral to a graft. Therefore, data from these groups were combined. The body mass of female rats was $60 \%$ to $75 \%$ of the male value at the time of grafting and throughout the postoperative period. At 56 days postgrafting, the body mass of female rats was 248 $\pm 8.7 \mathrm{~g}$; the male value was $433 \pm 7.9 \mathrm{~g}$.

The mass of grafted and control muscles expressed in absolute terms for females were $60 \%$ to $65 \%$ of the values for males (Table 1). However, when muscle mass was expressed relative to body mass, there was no difference due to gender for EDL and SOL control muscles and grafts (Table 1).

The absolute length of EDL and SOL control muscles for females was $80 \%$ and $85 \%$ of the value for males, respectively (Table 1). There were no gender differences in absolute length of EDI. and SOL grafts. The length of EDL and SOL grafts in females was not different from control values, whereas the length of EDL and SOL grafts in males averaged $80 \%$ of control values. For males, the graft length/tibia length ratio for grafts was $75 \%$ and $85 \%$ of the length ratios for control EDL and SOL muscles, respectively (Table 1). No differences were evident in the length ratios of grafts compared to the length ratios of control muscles for females.

The mean fiber area of female EDI and SOI control muscles was $60 \%$ and $75 \%$ of values for male muscles, respectively. No differences due to gender were observed in the mean fiber area of EDL or SOL grafts (Figs. I and 2; Table 1). Therefore, when mean fiber area of grafts was expressed as a percentage of control value, female EDL grafts were $85 \%$ of control value, whereas male EDL grafts were $60 \%$ of control value. This gender difference was more pronounced for the SOL in which grafts were $80 \%(P<0.11$ and $40 \%$ of control values for females and males, respectively.

The number of fibers per square millimeter of cross-sectional area was consistent with the mean fiber area data. In control SOL muscles, there were $307 \pm 21$ fibers per square millimeter for females and $201 \pm 9$ libers for males. This gender difference was also observed in control EDL muscles, in which there were $401 \pm 18$ and $246 \pm 19$ fibers per square millimeter for females and males, re- 


\begin{tabular}{|c|c|c|c|c|}
\hline \multirow[t]{3}{*}{ Variable } & Control & Graft & Control & Graft \\
\hline & & & EDL muscle & \\
\hline & \multicolumn{2}{|c|}{ Female } & \multicolumn{2}{|c|}{ Male } \\
\hline Muscle mass (mg) & $131 \pm 3.6^{*}$ & $124=5.5^{\star}$ & $217 \pm 9.2$ & $190 \pm 20.6$ \\
\hline $\begin{array}{l}\text { Tissue mass ratio } \\
\text { (mg/g body mass) }\end{array}$ & $0.52 \pm 0.011$ & $0.51 \pm 0.031$ & $0.51 \pm 0.023$ & $0.45 \pm 0.032$ \\
\hline Muscle length (mm) & $30 \pm 1.0^{\star}$ & $28 \pm 1.5$ & $38 \pm 0.3$ & $28 \pm 1.5 \dagger$ \\
\hline $\begin{array}{l}\text { Muscle length/ } \\
\text { tibia length }(\mathrm{mm} / \mathrm{mm})\end{array}$ & $0.88 \pm 0.029$ & $0.78 \pm 0.046$ & $0.93 \pm 0.015$ & $0.69 \pm 0.040 \dagger$ \\
\hline Mean fiber area $\left(\mu \mathrm{m}^{2}\right)$ & $2120 \pm 107.4^{*}$ & $1812 \pm 66.0 \dagger$ & $3645 \pm 341.4$ & $2148 \pm 150.6 \dagger$ \\
\hline \multicolumn{5}{|l|}{ Muscle composition } \\
\hline Type $1(\%)$ & $4 \pm 1.0$ & $11 \pm 4.8$ & $4 \pm 0.7$ & $5 \pm 1.0$ \\
\hline Type $2 a(\%)$ & $33 \pm 2.2$ & $29 \pm 8.1$ & $37 \pm 3.5$ & $27 \pm 2.1 \dagger$ \\
\hline \multirow[t]{3}{*}{ Type $2 b(\%)$} & $63 \pm 2.5$ & $60 \pm 10.3$ & $59 \pm 3.5$ & $67 \pm 1.7$ \\
\hline & & & SOL muscie & \\
\hline & \multicolumn{2}{|c|}{ Female } & \multicolumn{2}{|c|}{ Male } \\
\hline Muscle mass (mg) & $117 \pm 3.9^{*}$ & $114 \pm 10.2^{*}$ & $197 \pm 6.4$ & $181 \pm 26.1$ \\
\hline $\begin{array}{l}\text { Tissue mass ratio } \\
\text { (mg/g body mass) }\end{array}$ & $0.47 \pm 0.018$ & $0.48 \pm 0.036$ & $0.45 \pm 0.012$ & $0.42 \pm 0.050$ \\
\hline Muscle length $(\mathrm{mm})$ & $25 \pm 1.0^{*}$ & $23 \pm 1.2$ & $29 \pm 0.7$ & $25 \pm 1.0 \dagger$ \\
\hline $\begin{array}{l}\text { Muscle length/ } \\
\text { tibia length }(\mathrm{mm} / \mathrm{mm})\end{array}$ & $0.72 \pm 0.009$ & $0.66 \pm 0.035$ & $0.70 \pm 0.025$ & $0.61 \pm 0.023 \dagger$ \\
\hline Mean fiber area $\left(\mu \mathrm{m}^{2}\right)$ & $2907 \pm 115.4^{*}$ & $2290 \pm 296.9$ & $3961 \pm 171.2$ & $1602 \pm 94.9 \dagger$ \\
\hline \multicolumn{5}{|l|}{ Muscle composition } \\
\hline Type $1(\%)$ & $85 \pm 2.2$ & $94 \pm 2.6 \dagger$ & $85 \pm 2.2$ & $84 \pm 3.1$ \\
\hline Type $2 a(\%)$ & $8 \pm 1.3$ & $5 \pm 2.0$ & $13 \pm 2.0$ & $8 \pm 3.1$ \\
\hline Type $2 b(\%)$ & $6 \pm 1.3^{*}$ & $1 \pm 0.9 \dagger$ & $2 \pm 0.8$ & $7 \pm 4.0$ \\
\hline
\end{tabular}

:Significantly different $(P<0.05)$ from male value; $\uparrow$ significantly different $(P<0.05)$ from control value. Numbers of muscles: control ( $\mathrm{P}=10)$; graft $(n=6$ except maie soleus $n=5)$.

spectively. The number of fibers per square millimeter for muscle grafts did not differ due to gender: The female EDL grafts had $429 \pm 9$ and male EDL grafts had $376 \pm 48$ fibers; the female SOL had $342 \pm 42$ and the male SOL had $350 \pm 9$ fibers.

The fiber type composition of grafts was analyzed as a percentage by number and percentage by area. There were no differences between either analytical approach; we have presented the data as percentage by area (Table 1). With one exception (control SOL \% 2b fibers) there were no signilicant differences in the fiber composition of each type of muscle due to gender in graft or control muscles. There were no major differences in the fiber type profiles of female and male EDL and SOL grafts compared to their respective control muscles.

\section{Discussion}

The differences due to gender observed in length and mass of control EDL and SOL muscles correlated positively with gender differences in whole body mass. Indicative of this was the lack of gender differences when muscle mass was normalized to body mass and when muscle length was nor- malized to tibia bone length. Hormones such as growth hormone and androgens have been implicated in the regulation of body growth. ${ }^{11.13}$ The average plasma level of growth hormone does not differ between male and female rats. ${ }^{11}$ However, the diurnal pattern of secretion begins to differ coincident with an increase in the growth rate of males relative to females. Peak levels of hormone secretion were lower in female than male rats, but occurred more frequently during a 24-hour period.

There is some question as to whether limb muscles are sensitive to hormone regulation. Kochakian and colleagues ${ }^{17}$ found no effects of castration on growth rate of limb muscles and concluded that these muscles were not androgen-sensitive. Using the model of synergist tenotomy, Goldberg and colleagues $^{14}$ concluded that compensatory growth of skeletal muscle was hormone-independent. Mackova and Inik ${ }^{18}$ found no differences between female and male rats in the ability of muscles to hypertrophy. Carlson and colleagues transplanted the SOL into the bed of the hormonesensitive levator ani muscle in an attempt to induce hormone sensitivity on the SOL, but found none. 

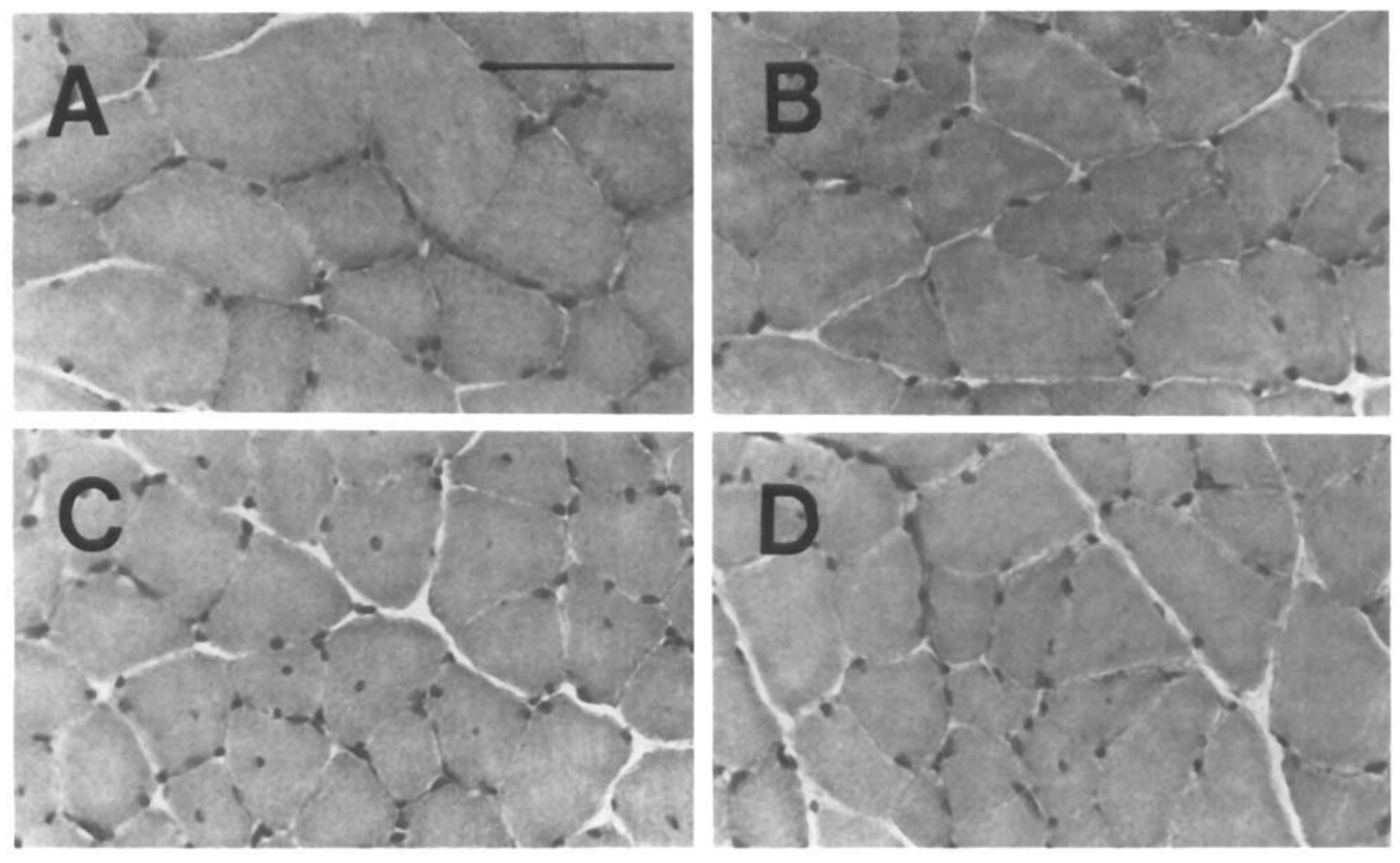

FIGURE 1. Photomicrographs of hematoxylin and eosin-stained transverse sections of (A) male control EDL, (B) female control EDL, (C) male 56-day EDL graft, and (D) female 56-day EDL graft. Bar represents $100 \mu \mathrm{m}$.

They also discovered the hormone sensitivity of the levator ani muscle was myogenic, so the location of the SOL graft likely had no effect on the androgen sensitivity of this muscle.

Minor differences in fiber composition between graft and control muscles were noted only for female SOL and male EDL. Although these differences were statistically significant, they are probably of limited biological significance. Recruitment patterns have been shown to influence the fiber type composition of a muscle. ${ }^{2,22}$ Our data are consistent with the grafts being innervated and recruited in a manner not different from control muscle.

To evaluate the regenerative capacity of different muscles from different genders, comparisons must be made when graft values are considered as a percentage of the particular control muscle value, and not in absolute terms. With respect to mass and fiber composition of grafts, there is no difference in regenerative capacity due to gender. However, the length and fiber cross-sectional area of grafts in females consistently attained a greater percentage of control value than in males.

In control EDL and SOL muscles, the larger fiber cross-sectional area of male muscles was directly related to the larger muscle mass of males compared to females (Figs. 1 and 2; Table 1). However, our data indicated the relations due to gender between mass and cross-sectional area which existed in control muscles were uncoupled in grafts. This raises the possibility that there is a maximum regenerative capacity for single muscle fibers. The magnitude of this capacity is likely mediated by factors common to both genders, including blood flow, innervation, stretch, and chronic recruitment patterns. The reason for the uncoupling between mass and fiber area remains to be elucidated. It is possible that compared to control muscles, grafts have more muscle fibers, longer muscle fiber lengths, more extracellular material (Figs. 1 and 2), or some combinations of these.

We observed no substantial differences in the regenerative capacity of EDL and SOL muscles grafted while leaving the nerve intact during the grafting operation. The degree of restoration of structure and function of standard grafts of $\mathrm{EDL}^{8}$ and SOL muscles ${ }^{10,23}$ has been variable, and the SOL has not regenerated to the same relative ex- 

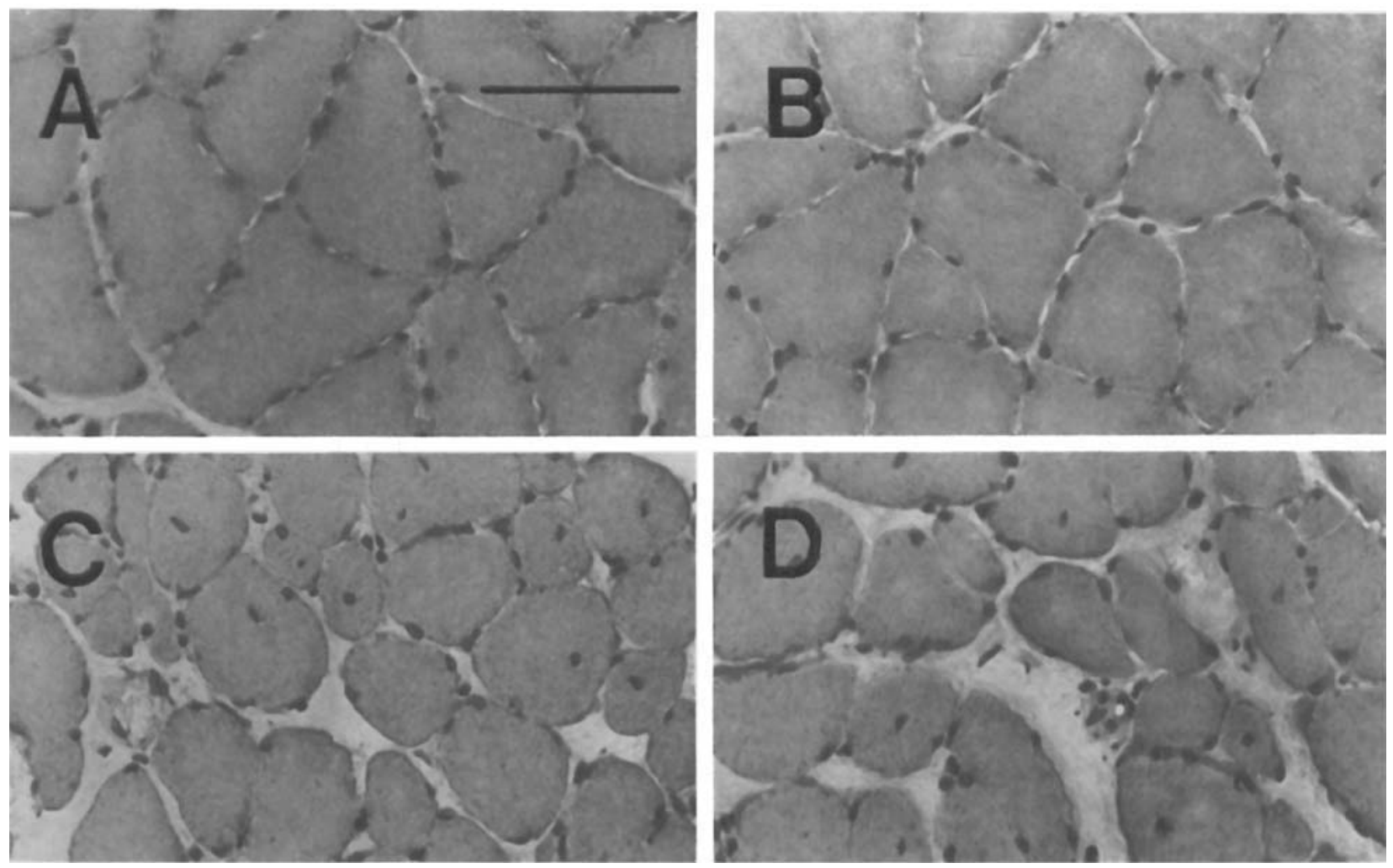

FIGURE 2. Photomicrographs of hematoxylin and eosin-stained transverse sections of (A) male control SOL, (B) female control SOL, (C) male 56-day SOL graft, and (D) female 56-day SOL graft. Bar represents $100 \mu \mathrm{m}$.

tent as the EDL. In standard grafting operations, the nerve is cut, and subsequent reattachment and reinnervation occur spontaneously. Differences between the EDL and SOL muscles in the angle of insertion of the primary motor nerve ${ }^{16}$ may account for differences in the degree of regeneration in standard grafts. It remains to be elucidated if this is due to a slower rate or a smaller amount of reinnervation of the standard SOL graft, compared to standard EDL grafts. Coan and Tomanek $^{10}$ have attributed the poor persistence of standard SOL grafts to lack of functional reinnervation. White and colleagues ${ }^{24}$ have reported the mass of SOL muscles grafted with a nervereimplant operative technique stabilized at $60 \%$ of control SOL muscle value, and that these grafts persisted through 112 days postgrafting. Our current data with nerve-intact grafts are consistent with an increased speed of reinnervation as well as a more widespread distribution of nerve fibers in nerve-intact grafts compared to standard grafts. ${ }^{7}$

\section{REFERENCES}

1. Ariano MA, Armstrong RB, Edgerton VR: Hindlimb muscle fiber populations of five mammals. J Histochem Cytochem 21:51-55, 1973

2. Barany $M$, Close $R I$ : The transformation of myosin in cross-innervated rat muscles. J Physiol (Lond) 213:455-474, 1971.

3. Brooke MH, Kaiser KK: Muscle fiber types: how many and what kind? Arch Neurol 23:369-379, 1970.

4. Carlson BM, Faulkner JA: The regeneration of skeletal muscle fibers following injury: a review. Med Sci Sports 15:187-198, 1983.

5. Carlson BM, Gutmann E: Regeneration in free grafts of normal and denervated muscles in the rat: morphology and histochemistry. Anat Rec 183:47-62, 1975.
6. Carlson BM, Herbrychova A, Gutmann E: Retention of hormonal sensitivity in free grafts of the levator ani muscles. Exp Neurol 83:94-107, 1979.

7. Carlson BM, Hnik P, Tucek S, Vejsada R, Bader DM, Faulkner JA: Comparison between grafts with intact nerves and standard free grafts of the rat extensor digitorum longus muscle. Physiol Bohemoslov 30:505-513, 1981.

8. Carlson BM, Wagner KR, Max SR: Reinnervation of rat extensor digitorum longus muscles after free grafting. Muscle Nerve 2:304-307, 1979.

9. Chayen J, Bitensky L, Butcher RG, Poulter LW: Practical Histochemistry. London, John Wiley \& Sons, 1973, pp 24-25, 177-187.

10. Coan MR, Tomanek RJ: The growth of regenerating soleus 
muscle transplants after ablation of the gastrocnemius muscle. Exp Neurol 71:278-294, 1981.

11. Eden S: Age- and sex-related differences in episodic growth hormone secretion in the rat. Endocrinology 105: $555-560,1979$

12. Faulkner JA, Markley JM Jr, McCully KK, Watters, CR, White TP: Characteristics of cat skeletal muscles grafted with intact nerves or with anastomosed nerves. Exp Neurol 80:682-696, 1983.

13. Goldberg AL: Work-induced growth of skeletal muscle in normal and hypophysectomized rats. Am J Physiol 231: 1193-1198, 1967.

14. Goldberg AL, Etlinger JD, Goldspink DF, Jablecki C: Mechanism of work-induced hypertrophy of skeletal muscle. Med Sci Sports 7:248-261, 1975.

15. Goodrick CL: Effects of long-term voluntary wheel exercise on male and female Wistar rats. Longevity, body weight, and metabolic rate. Gerontology 26:22-33, 1980.

16. Greene E: Anatomy of the Rat. New York, Hafner, 1968, pp 322-323.

17. Kochakian CD, Tillotson C, Austin J, Dougherty E, Haag V, Coalson R: The effect of castration on the weight and composition of the muscles of the guinea pig. Endocrinology
$58: 315-326,1956$

18. Mackova E, Hnik P: Time course of compensatory hypertrophy of slow and fast rat muscles in relation to age. Physiol Bohemoslov 21:9-17, 1972.

19. Marascuilo LA: Statistical Methods for Behavioral Science Research. San Francisco, McGraw-Hill, 1971, pp 312-314.

20. Nachlas M, Tsou MK, de Souza E, Cheng C, Seligman AM Cytochemical demonstrations of succinate dehydrogenase by the use of a new p-nitrophenyl substituted ditetrazol. $J$ Histochem Cytochem 5:420-436, 1957.

21. Peter JB, Edgerton VR, Gillespie CA, Stemple KE: Metabolic profiles of three fiber types of skeletal muscles of guinea pigs and rabbits. Biochemistry 11:2627-2633, 1972.

22. Salmons S, Sreter FA: Significance of impulse activity in the transformation of skeletal muscle types. Nature 263:30-34, 1976.

23. Schmalbruch $\mathrm{H}$ : Regeneration of soleus muscles of rat autografted in toto as studied by electron microscopy. Cell Tissue Res 177:159-180, 1977.

24. White TP, Villanacci JF, Morales PG, Segal SS, Essig DA: Exercise-induced adaptations of rat soleus muscle grafts. $J$ Appl Physiol: Respirat. Environ. Exercise Physiol. 56: 1325-1334, 1984. 